\title{
Aux Olympiades du progrès : les ouvriers italiens aux expositions universelles au XIXe siècle
}

Olympic games of the progress: Italian workers at the $19^{\text {th }}$ century international exhibitions

\section{Anna Pellegrino}

\section{Q OpenEdition}

\section{Journals}

Édition électronique

URL : http://journals.openedition.org/dht/322

ISSN : 1775-4194

Éditeur :

Centre d'histoire des techniques et de l'environnement du Cnam (CDHTE-Cnam), Société des élèves du CDHTE-Cnam

\section{Édition imprimée}

Date de publication : 1 décembre 2009

Pagination : 113-130

ISBN : 978-2-9530779-4-0

ISSN : 0417-8726

\section{Référence électronique}

Anna Pellegrino, «Aux Olympiades du progrès : les ouvriers italiens aux expositions universelles au XIXe siècle », Documents pour l'histoire des techniques [En ligne], $18 \mid 2^{\mathrm{e}}$ semestre 2009, mis en ligne le 06 avril 2011, consulté le 07 septembre 2020. URL : http://journals.openedition.org/dht/322 


\title{
Aux Olympiades du progrès : les ouvriers italiens aux expositions universelles au $\mathrm{XIX}^{\mathrm{e}}$ siècle
}

\author{
Anna Pellegrino \\ Université de Padove
}

\begin{abstract}
RésumÉ
Cet article présente les premiers résultats d'une recherche sur la participation des ouvriers italiens aux expositions universelles $d u X I X X^{e}$ siècle. II s'agit d'un phénomène qui a été peu analysé, mais qui a revêtu des dimensions de masse et connu une continuité considérable au cours du temps. Y contribuèrent des organismes publics, des entrepreneurs, les organisateurs mêmes des expositions, mais aussi des ouvriers, chacun avec des buts différents, soit didactiques et de perfectionnement professionnel, soit d'implication politique et culturelle dans l'idéologie du progrès et de la modernisation qui sous-tendait les expositions. Les comptes-rendus écrits par les ouvriers à la fin de leur voyage représentent une source directe pour les historiens, une forme d'écriture "populaire " qui permet de connaître les opinions de ces visiteurs, leurs impressions générales, mais aussi d'analyser leur culture, leurs compétences techniques et leur langage. Dans cet article, nous approfondissons quelques problèmes méthodologiques relatifs à l'usage de ces archives et présentons une première sélection des sujets traités dans les comptes-rendus des ouvriers, à savoir le rapport entre l'innovation technique, le vécu du progrès technique et les répercussions de celui-ci sur l'emploi et les rythmes de travail. La mémoire du métier traditionnel, la tension - typique des rencontres internationales - entre nationalisme et internationalisme sont aussi analysées. Finalement, une attention particulière est réservée au rôle spécifique du voyage et de la rencontre avec la métropole parisienne dans l'expérience que vivent les ouvriers lors des expositions.
\end{abstract}

Résumés et mots clés en anglais sont regroupés en fin de volume, accompagnés des mots clés français

" La stupide exposition m'est devenue tellement odieuse que vraiment je me réjouis de ne pas la voir »'.

A insi s'exprimait Friedrich Engels dans une lettre à Karl Marx le 9 septembre 1862, à l'occasion de la seconde exposition universelle de Londres.

Si les expositions universelles du XIXe siècle ont constitué un spectacle fascinant pour un large public, si leur forte popularité a été soulignée par nombre d'études, nous connaissons moins bien l'accueil

1 « La stupida esposizione, attraverso il sentito dire, mi è diventata talmente odiosa, che davvero mi rallegro di non vederla »; lettre de Friedrich Engels à Karl Marx, 9 septembre 1862, dans Marx-Engels, Carteggio, vol. 4, traduit en italien par Sergio Romagnoli, Roma, Editori Riuniti, 1972, p. 130 ; " everything l've heard about that idiotic exhibition ... has made me hate it so much that I'm downright glad I shan't be seeing it 1 , Marx-Engels correspondance 1862, Marx/Engels collected works (MECW), Londres/New York, Progress Publishers of the Soviet Union/Lawrence \& Wishart/International Publishers, 1985, vol. 41, p. 414, transcrit dans Marx and Engels Internet Archive (MIA), 2000. différencié réservé à ces dispositifs complexes. Non seulement les intellectuels socialistes ont pu être critiques, mais il semble que les publics populaires aient été parfois désorientés, comme en témoigne cette remarque d'un ouvrier italien en 1906 :

\footnotetext{
" Les images qui nous en recevons sont variées et innombrables, au point que la somme de celles-ci et leur réflexion dans l'esprit seraient à comparer, s'il est permis, à la sensation psychologiquement opposée mais également tumultueuse décrite dans « Une tempête sous un crâne » dont Victor Hugo parle magistralement, dans un de ses travaux plus connus $1^{2}$.
}

\footnotetext{
2 Relazione di Renzi Giulio, meccanico, aggiustatore, montatore della fabbrica di automobili Florentia, Archives municipales de Florence [ensuite AM Florence], Cerimonie, Festeggiamenti, Esposizioni, cart. 3, n. 5050 (" le imagini [sic] che se ne ricevono, sono variate ed innumerevoli, si che la somma di queste, e la loro riflessione nell'intelletto, si potrebbe paragonare, se il raffronto fosse lecito, alla sensa-
} 
Cet extrait fait partie d'un compte-rendu de visite à l'exposition internationale de Milan cette même année. C'est cette documentation très vaste et en grande partie inédite des visites ouvrières aux expositions dans la deuxième moitié du XIXe siècle et début du $X X X^{e}$ siècle que nous nous proposons d'étudier. II s'agit d'une forme d'écriture « populaire » difficile à analyser, mais susceptible de révéler les modes de réception du message véhiculé par les expositions - un thème peu traité jusqu'à présent.

L'accueil populaire pouvait aller de l'adhésion enthousiaste à la critique radicale, mais il mettait toujours en jeu le rapport des travailleurs avec les nouveautés techniques. Sur ce point, notre approche s'inscrit dans un courant de recherche en plein essor. Après avoir été étudiées dans leurs aspects économiques, urbanistiques et architecturaux, les expositions universelles sont aujourd'hui considérées comme des lieux de diffusion des connaissances scientifiques et techniques, à l'origine de la création de réseaux commerciaux et d'infrastructures, participant aux processus d'intercommunication, d'échange culturel et de mondialisation ${ }^{3}$. Ces manifestations ont, en effet, mobilisé l'opinion publique dans des proportions surprenantes si l'on songe, par exemple, qu'à l'exposition parisienne de 1900, sont enregistrées plus de cinquante millions d'entrées payantes. Elles constituent un média

zione psicologicamente opposta, ma egualmente tumultuaria descritta in quella 'Tempesta in un cranio' di cui Victor Hugo, magistralmente parla, in uno dei suoi lavori più noti »). 3 Sur les expositions universelles en général, Paul Greenhalgh, Ephemeral vistas: the expositions universelles, Great Exhibitions and World's Fairs, 1851-1939, Manchester, Manchester University Press 1988 ; Le livre des expositions universelles 1851-1989, Paris, Édition des arts décoratifs, Hersher, 1983 ; Linda Aimone, Carlo Olmo, Le Esposizioni Universali 1851-1900, Turin, 1990 ; Brigitte Schroeder-Gudehus, Anne Rasmussen, Les fastes du progrès. Le guide des Expositions universelles 18511922, Paris, Flammarion, 1992 ; Robert W. Rydell, Nancy E. Gwinn (dir.), Fair Representation : World's Fairs and the Modern World, Amsterdam, VU University Press, 1994. Sur les expositions comme phénomènes précurseurs des processus de communication et de globalisation, Alexander C.T. Geppert, Massimo Baioni, Esposizioni in Europa fra Otto e Novecento. Spazi, organizzazione, rappresentazioni, numéro monographique de Memoria e Ricerca, 17/2004; Alberto Abruzzese, Lessico della comunicazione, Rome, Meltemi, 2003, pp. 170-183; Armand Mattelart, L'invention de la communication, Paris, La Découverte, 1997, pp. 131-152 ; Peter Sloterdijk, Le palais de cristal : à l'intérieur du capitalisme planétaire, Paris, Maren Sell Éditeurs, 2006. de masse, de portée considérable, alors que le développement des moyens de communication est encore embryonnaire. Nous voudrions précisément interroger la sociologie des publics mobilisés et notamment, les modes de participation des milieux populaires, et en particulier ouvriers.

Indubitablement, les classes dirigeantes ont joué un rôle hégémonique dans l'organisation et la présentation des expositions. Cependant, une série d'études récentes a partiellement révisé l'implication du monde du travail, en particulier celle des travailleurs eux-mêmes, en en montrant l'ampleur et le grand intérêt. L'usage d'envoyer les ouvriers aux expositions à des fins pédagogiques ou de promotion remonte à la première exposition nationale française, organisée en 1798 au Champ de Mars ${ }^{4}$.

À l'occasion de la Great Exhibition of the works of industry of all nations de Londres en 1851, le prince Albert avait expressément déclaré que ce type de manifestation visait non seulement à fournir un lieu d'échange et de comparaison pour les industriels et les producteurs, mais aussi et surtout à élever le niveau de connaissances et d'intérêt du peuple anglais pour les produits de la science et des "arts industriels". Cet intérêt aurait des effets bénéfiques sur le goût, l'éducation et la morale des classes populaires (petite bourgeoisie, artisanat et boutique, prolétariat industriel) $)^{5}$. Les tentatives et les stratégies pour susciter des formes de participation, lors des expositions, furent nombreuses : elles allaient de la réduction du prix d'entrée selon les heures ou les jours de la semaine, à des tarifs spéciaux pour le voyage et l'entrée gratuite réservée à des catégories de personnes, jusqu'à la prise en charge complète du voyage et du séjour pour des groupes d'ouvriers triés sur le volet. Cette dernière supposait, en premier lieu, une sélection forte des ouvriers à envoyer à l'exposition. Elle comportait, en outre, l'adoption de divers mécanismes de contrôle, de recherche du consensus et de détermination fonctionnelle des buts de la visite. Elle prévoyait, enfin, la rédaction d'un compterendu à l'issue de la visite, matériel très intéressant pour évaluer comment les ouvriers percevaient les expositions et exprimaient leurs impressions. Ces comptes rendus, qui ont été publiés et sont rapidement entrés dans le discours public sur les expositions, sont l'objet de cet article. Ils

4 Sigmund Engländer, Geschichte der Französischen Arbeiter - Associationen, Hambourg, Hoffman und Campe, 1864, I, pp. 51-53, cité par Walter Benjamin, Parigi Capitale del XIX secolo. I "passages" di Parigi, Rolf Tiedmann dir., Turin, Einaudi, 1982, p. 240.

5 Jeffrey A. Averbach, The Great Exhibition of 1851: a nation on display, Yale University Press, New Haven, 1999, pp. 9-15 et 129-137. 
manifestent un intérêt pour la participation effective d'un public large et " populaire " aux expositions, encore peu présent dans l'historiographie alors que les études culturelles sur les expositions ont connu un réel renouveau'.

Dès l'origine, nous l'avons dit, les expositions se sont donné pour objectif de rallier les classes populaires et travailleuses, comme le montrent diverses études qui soulignent la capacité des expositions à attirer, à " distraire ", à " amuser " le public ouvrier, tout en occultant et en masquant les conflits internes au système productif dont elles célébraient les fastes ${ }^{7}$.

La place centrale des enjeux éducatifs ef explicitement didactiques des expositions est au cœur d'un ouvrage récent et important sur le phénomène, ouvertement consacré, comme l'indique son sous-titre, aux expositions comme " didactic phenomenon $\|^{8}$. D'après son auteur, les expositions auraient fonctionné comme un canal de médiation sociale et culturelle : " comme un intermédiaire entre la culture d'en bas et la culture d'en haut, entre les élites, les classes moyennes et classes populaires, et d'une part entre le commerce, l'industrie, la technique, la science et l'art, d'autre part l'expérience vécue de tout un chacun 11 .

Beaucoup plus rares sont les analyses des

6 Les expositions ont été analysées soit comme précurseurs des processus de globalisation, soit comme événements centraux dans l'émergence des processus d'artificialisation et de " virtualisation " qui caractérisent notre société contemporaine (le " visual and spatial turn ») ; voir Alexander C.T. Geppert, « Città brevi: storia, storiografia e teoria delle pratiche espositive europee, 1851-2000 ॥, dans A.C.T. Geppert, M. Baioni éd., Esposizioni in Europa, op. cit., pp. 7-18.

7 La version la plus récente de cette approche a été produite en France grâce aux travaux de Madeleine Rebérioux, « Les ouvriers et les expositions universelles de Paris au XIXe siècle 1 , dans Le livre des expositions universelles, op.cit, pp. 197-208 id., « Mise en scène et vulgarisation : l'exposition universelle de 1889 I), Le mouvement social ,149, octobre-décembre,1989, en particulier l'essai introductif "Au tournant des expos ॥, pp. 3-13; nº spécial de La revue de l'économie sociale (XIX, 1990), consacré au thème Les expositions universelles à Paris. Sur l'exposition parisienne de 1867 et sur la politique d'inclusion de classes populaires, Volker Barth, « Displaying normalisation: The Paris universal exhibition of 1867 ॥, Journal of historical sociology, vol. $20, n^{\circ} 4,2007$, pp. 462-485.

8 Pieter van Wesemael, Architecture of instruction and delight. A socio historical analysis of World Exhibitions as a didactic phenomenon (1798-1851-1970), Rotterdam, 010 Publishers, 2001.

9 « As an intermediary between high and low culture, between upper, middle and lower class, and between trade, industry, technology, science and art on the one hand, and the lay person's more direct world of experience on the other "), ibid., p. 17. voyages ouvriers et des comptes-rendus respectifs qui conduisent à une reconsidération de la mentalité et de la culture ouvrières, ou plus exactemement entre les classes supérieures, moyennes et inférieures de la société.

Une première difficulté est de définir les frontières précises de la catégorie des " ouvriers ॥. En Italie, au moment de l'unification nationale (1861), a lieu un débat intense au sein des organisations de travailleurs pour définir l'usage de ce terme et les figures sociales auxquelles il doit se référer. L'extension sémantique du terme est alors assez marquée. Le mot " ouvrier " rassemble en effet des figures très différentes : des travailleurs à domicile aux apprentis des ateliers artisanaux, des travailleurs subalternes des entreprises industrielles aux travailleurs des entreprises commerciales et des services jusqu'aux artisans et aux petits entrepreneurs.

Il est évident qu'une telle définition, susceptible de glissements sémantiques considérables, durant les années qui vont jusqu'à la Première guerre mondiale ${ }^{10}$, répondait à des buts normatifs et "politiques " plutôt qu'à des critères d'enquête sociologique. En analysant les sources, une dialectique persistante apparaît entre l'identité professionnelle spécifique et l'identité collective plus générale résumée dans le terme " ouvrier ». Cette dernière est utilisée surtout en raison des problèmes d'ordre général qui vont des aspects économique-syndicaux jusqu'aux choix et aux orientations politiques de base (nationalisme, pacifisme etc.), ou des problèmes particuliers relatifs aux modèles comportementaux et " moraux $\|^{11}$, jusqu'à ceux de la sphère quotidienne et familiale.

10 Une délégation d'ouvriers (« operaj ») florentins participe à l'exposition universelle de Londres de 1851: ils sont en réalité tous, sans exception, des entrepreneurs ; voir « Rapporti sulla spedizione degli operaj toscani alla Grande Esposizione di Londra letti alla Reale Accademia dei Georgofili dai soci ordinario Cavalier Francesco Bonajni e corrispondente Angiolo Venni I), dans Atti dei Georgofili, Nuova serie, vol. I, Florence, Tip. Galileiana, s.d. Ensuite le terme est utilisé dans les sources pour désigner des rôles très différents dans le monde du travail. Le contexte social d'appartenance est aussi déterminant. Par exemple, les 133 « ouvriers intellectuels » florentins envoyés à l'exposition de Milan en 1906 sont, dans leur grande majorité, des artisans. Les ouvriers romains qui sont à Paris en 1878 et en 1889 sont aussi dans la majorité des artisans (couturiers, imprimeurs, etc.). Par contre, les ouvriers milanais qui se rendent à Paris en 1900 sont plus proches de la figure sociale de l'ouvrier d'usine.

11 Une grande partie des sociétés ouvrières italiennes de la seconde moitié du XIXe siècle établissent dans leurs statuts des règles " morales " précises, comme les devoirs envers la patrie, l'honnêteté, la conduite dans le milieu familial, etc. 


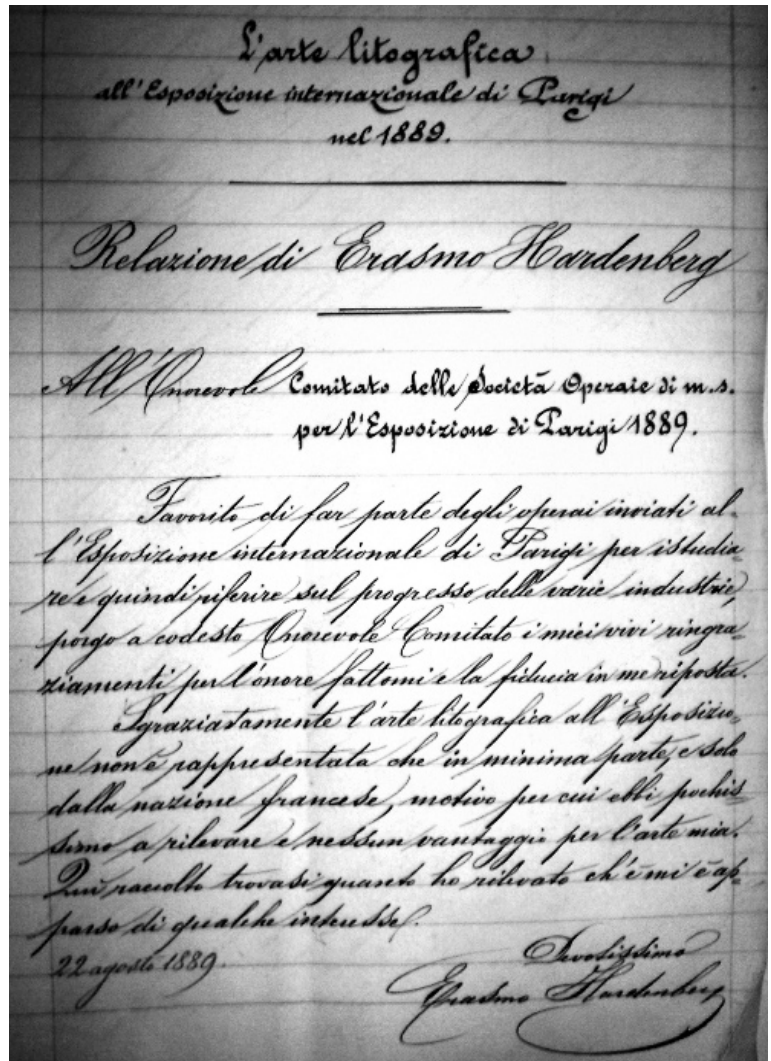

fig. 1 - Première page du compte rendu d'Erasmo Hardenberg à l'exposition Universelle de Paris en 1889, "L'arte litografica all'Esposizione Internazionale di Parigi nel 1889", Archivio civico del Comune di Milano, Esposizioni e Fiere, Esposizione di Parigi 1889, cart. n. 3.

Les comptes-rendus des ouvriers aux expositions font souvent référence, au-delà des sujets professionnels spécifiques, à ces problèmes plus globaux.

À ce sujet, nous devons à Jacques Rancière et Patrick Vauday un essai pionnier. Ces auteurs ont placé au centre de leur recherche le thème de la mentalité ouvrière ainsi que des problèmes plus larges, notamment les conceptions liées au genre. Néanmoins, ils se sont surtout intéressés au rapport avec les "philosophies politiques ॥ qui contribuent de manière déterminante aux attributions identitaires du monde ouvrier de l'époque ${ }^{12}$. En Italie également, plusieurs études ont porté sur des cas de voyages et d'écritures ouvrières relatives aux expositions ${ }^{13}$.

12 Jacques Rancière et Patrick Vauday, «En allant à l'expo : l'ouvrier, sa femme et les machines "I, Les révoltes logiques, n० 1, 1976, pp. 5-22.

13 Concernant le cas italien, une série d'études abordent aussi des thématiques spécifiques : sur le rôle subordonné attribué aux classes populaires à l'exposition italienne de 1911, par exemple, Antonio Parisella, "Fuori dalla scena: le classi popolari e l'Esposizione del 1911 11, dans Gianna Piantoni éd., Roma 1911, Rome, De Luca, 1980, pp. 53-66 ; concernant un cas individuel, Augusta Molinari, « Cronaca di
Toutefois, dans l'ensemble, on doit signaler l'absence d'analyse du phénomène dans toute sa complexité, autrement dit dans ses liaisons avec les différentes instances politiques, sociales et culturelles qui soit en jeu sur la scène des expositions, à commencer par l'écriture ouvrière elle-même.

\section{Les comptes rendus des ouvriers: quelques aspects méthodologiques}

Les comptes rendus des ouvriers se prêtent à différentes lectures et interprétations. Je me concentrerai sur les thématiques les plus fréquentes et distinctives de leur discours. Manié avec toute la prudence méthodologique qui s'impose, ce type de source révèle, de manière directe et indirecte, certains traits caractéristiques de la culture populaire et ouvrière.

La source principale de la recherche est constituée par les comptes-rendus des travailleurs italiens rédigés lors de leur visite à l'Exposition universelle de Londres en 1851 et à celles qui suivirent jusqu'à l'exposition de Paris en 1900. Le matériel à notre disposition se compose d'une documentation très vaste sur les initiatives prises par les principales villes italiennes (Milan-Turin-Rome-Florence-Bologne) pour financer les voyages des ouvriers dans les villes sièges de l'exposition. Ces récits sont rédigés sous les formes les plus diverses et expriment efficacement l'étonnement et l'enthousiasme des ouvriers face aux plus récentes merveilles du progrès. Les nouvelles machines et les découvertes de la technologie sont qualifiées de "fées » ou de « monstrueux mécanismes ».

On doit distinguer au moins deux types : les comptes-rendus édités et publiés à l'époque, souvent prédisposés dès l'origine dans ce but et donc contrôlés en vue de leur usage public, et les comptes-rendus envoyés aux comités promoteurs pour justifier et relater la visite achevée, qui par contre ont été produits et sont conservés dans les archives avec un degré moindre de réélaboration et de " manipulation ».

Les sources se présentent sous une configuration particulière. Ce sont des documents individuels qui expriment l'originalité du caractère, du métier et du style de leur auteur. En même temps, ellesse présentent réunies en corpus, car elles ont été conservées ou publiées sous forme de collections qui rassemblent les comptes rendus du même groupe d'ouvriers envoyés

un'esperienza memorabile. La visita di un operaio genovese all'esposizione internazionale di Torino del 1911 1), Ventesimo Secolo, a. I, n. 1, janvier-avril 1991, pp. 205-224 ; Anna Pellegrino, Operai intellettuali, Lavoro, tecnologia e progresso all'Esposizione di Milano, 1906, Manduria, Lacaita, 2008.

116 Documents pour l'histoire des techniques $\mathrm{n}^{\circ} 18$ - décembre 2009 


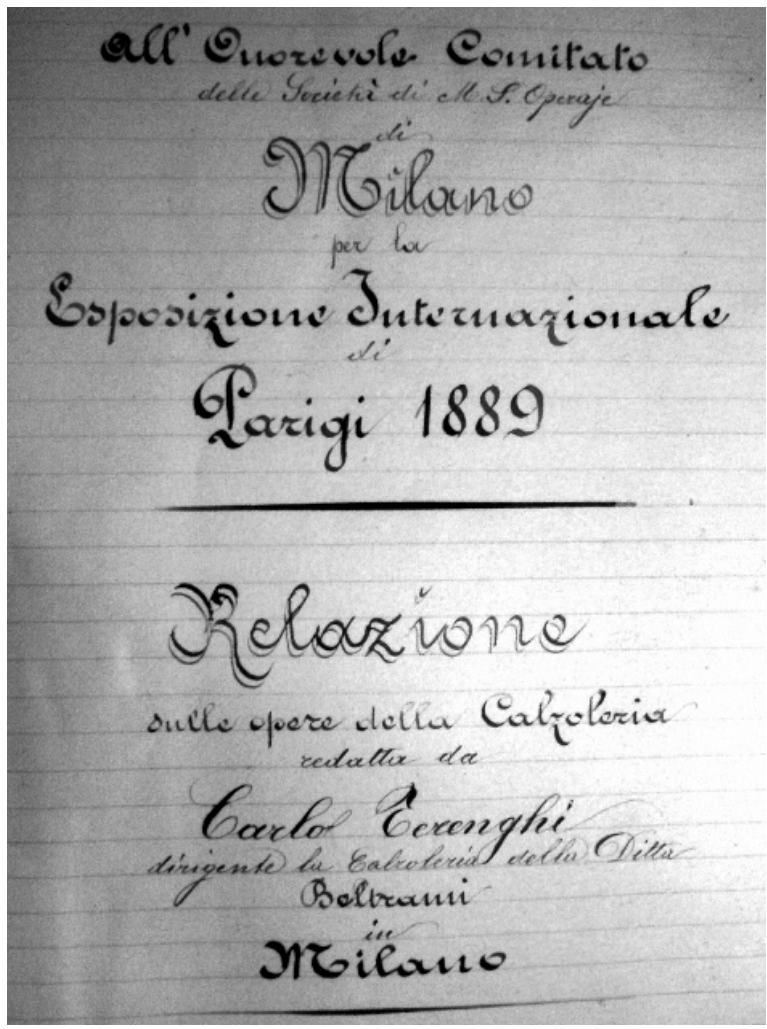

fig. 2 - Frontispice du compte rendu de Carlo Tereghi à I'Exposition universelle de Paris en 1889, "Relazione sulle opere della Calzoleria redatte da Carlo Terenghi dirigente la calzoleria della Ditta Beltrami in Milano", Archivio civico del Comune di Milano, Esposizioni e Fiere, Esposizione di Parigi 1889, Cart. n. 3.

aux expositions. De plus, elles illustrent l'expérience commune vécue et un même objet de description et représentation, par delà la conjoncture, le moment historique et les caractéristiques originales de chaque exposition.

En analysant les contenus des ces comptes rendus, il faut en outre considérer que les promoteurs, les bailleurs de fonds et les accompagnateurs conditionnaient de manière évidente, et parfois déterminante, le but, les objectifs et la modalité même du déroulement des visites, en exerçant un contrôle plus ou moins direct sur la conduite de la visite et sur le résultat final (la rédaction du récit).

Finalement, par-delà leurs contenus, les comptes rendus des ouvriers nous frappent par le langage employé. Le vocabulaire, le choix des termes, l'organisation du discours, la rhétorique et le style sont extrêmement variés. Construits parfois comme le traitement d'un sujet, circonscrit au travers du point de vue d'un métier particulier, ces rapports apportent non seulement des renseignements précieux sur l'objet, mais révèlent une articulation interne, une structure et une stratégie de communication d'un intérêt indéniable.

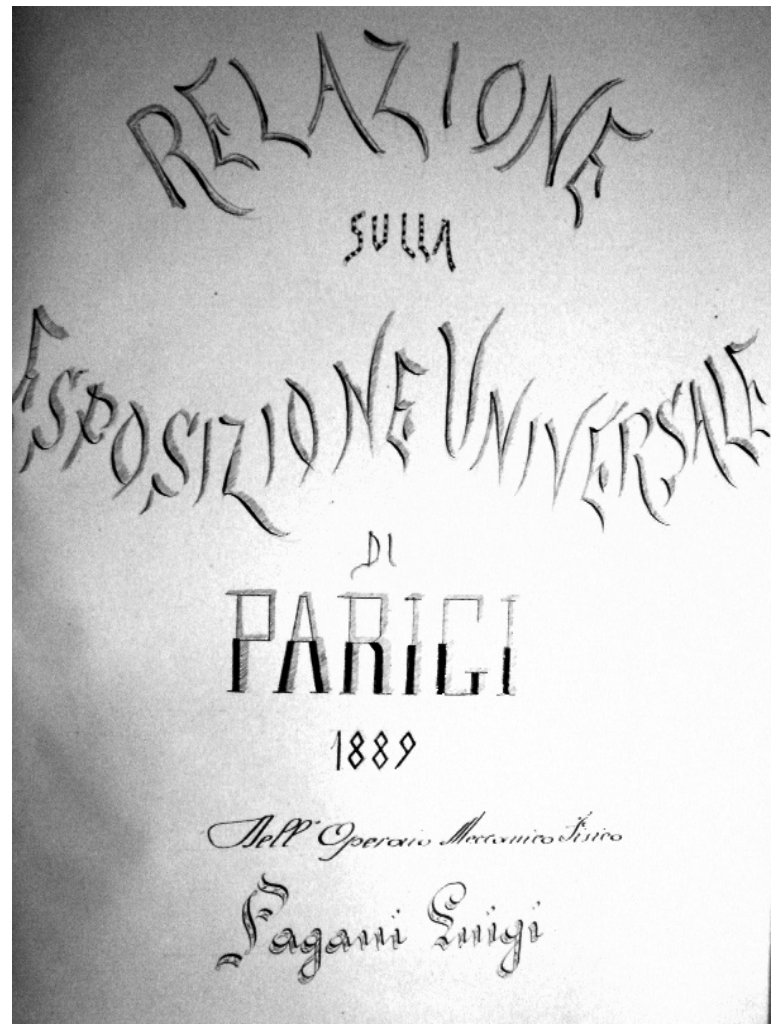

fig. 3 - Frontispice du compte rendu de Pagani Luigi à l'Exposition universelle de Paris en 1889, « Relazione Sulla Esposizione Universale di Parigi 1889 dell'Operaio Meccanico Fisico Pagani Luigi 1), Archivio civico del Comune di Milano, Esposizioni e Fiere, Esposizione di Parigi 1889, cart. n. 4.

Les comptes rendus relatifs aux expositions parisiennes sont conservés en grande partie dans les Archives de la Mairie et de la Chambre de commerce de Milan : il s'agit au total de 354 rapports, 121 relatifs à l'exposition de 1889 (Archives de la Mairie) et 233 concernant celle de 1900 (Archives de la Chambre de commerce). Pour l'exposition de 1900, nous disposons en outre, des rapports publiés à l'époque par les ouvriers de Turin envoyés à Paris dans le cadre d'une Commission ouvrière. Les rapports publiés sont au nombre de douze seulement, qui furent les seuls dignes d'être retenus sur un total de 68. Pour l'exposition de 1878, nous disposons de cinquante rapports édités à l'époque, qui concernent respectivement douze ouvriers de Rome envoyés par la Mairie et par la Chambre de commerce et trente-huit ouvriers de Turin envoyés par la Société promotrice de l'Industrie nationale de cette ville. En ce qui concerne l'exposition de Vienne en 1873, nous disposons de vingt rapports des ouvriers romains envoyés à l'exposition par la Société ouvrière de Rome, édités à l'époque. Nous bénéficions enfin pour l'exposition de Milan de 1906, de 133 rapports inédits des ouvriers florentins (Archives historiques de la Mairie de Florence) et de trente-cinq 
rapports des ouvriers de Bologne (Archives d'État de Bologne). On peut de plus ajouter à ces sources les comptes-rendus des ouvriers italiens aux expositions nationales (Milan 1881 - Turin 1884 et 1898 - Palerme 1892).

En plus de ces récits ouvriers, nous disposons de tous les documents administratifs relatifs à la sélection des ouvriers eux-mêmes, avec des informations sur leurs origines, leur culture et leur carrière professionnelle ; d'autres informations portent sur les critères qui ont orienté le choix des comptes-rendus pour les prix ou la publication. Nous avons également les matériaux préparatoires des commissions qui finançaient ces voyages, dont on peut tirer des renseignements sur les rapports avec les autorités locales de la ville siège de l'exposition et les autres associations ouvrières, sur l'organisation des voyages, sur les comités d'accueil, sur les logements où ils ont résidé, sur les lieux de socialisation, etc.

Les conditions particulières dans lesquelles se déroule le voyage de ces travailleurs, la prédétermination du but et de la fonction de leur visite, les formes mêmes du compte rendu influent certainement sur les thématiques choisies comme sujet central dans leurs écrits. II convient aussi d'attribuer un rôle important aux connaissances préalables des ouvriers et au bagage culturel spécifique, professionnel et général avec lequel ils arrivent à ce rendez-vous.

Les comptes rendus constituent donc pour I'historien un instrument pour analyser la façon dont se réalise dans l'écriture ouvrière l'intégration entre les connaissances et les conceptions préalables d'un côté, et l'impact de la rencontre avec la technique et les nouvelles configurations susceptibles de mettre en crise les cadres de référence précédents, de l'autre.

II reste à déterminer l'ampleur de la diffusion effective de ces rapports à l'intérieur du monde du travail de l'époque. Ces comptes-rendus restèrent pour une grande partie enfouis dans les archives. ॥ est donc difficile de déterminer dans quelle mesure les classes dirigeantes en ont pris acte pour introduire des améliorations dans leurs choix techniques et d'organisation du travail. Du côté ouvrier, on peut supposer que l'expérience restituée dans ces relations a constitué un puissant intermédiaire pour la circulation des idées relatives à la transformation des procédés de production et à la technique.

Trois thématiques présentes dans les comptesrendus ouvriers peuvent attirer l'attention. La première concerne la technique et le progrès. Le terme de «progrès » est l'un des mots les plus utilisés dans le cadre des expositions, c'est aussi l'un des traits marquants et chargés fortement d'un contenu idéologique de la tentative d'intégration des classes ouvrières par les classes dirigeantes ${ }^{14}$. Cette question n'est pas étrangère au langage des travailleurs de l'époque et à leurs organisations, et dans les comptes-rendus, elle vient s'articuler avec force à celle de la technique et à la comparaison entre innovation et tradition.

Le second thème a trait à l'identité nationale, à l'internationalisme etau pacifisme. Cette préoccupation appartient à la culture des travailleurs, tantl'organisation du mouvement ouvrier de l'époque revêt une forte orientation internationaliste et pacifiste; mais il s'agit aussi d'un champ de convergence intéressant avec une partie des cultures et des idéologies des classes dirigeantes, car l'internationalisme caractéristique du monde de la science constituait l'une des racines du phénomène des expositions, en dépit d'une tension dialectique avec le sentiment d'appartenance nationale.

La troisième orientation, enfin, concerne le rapport des travailleurs avec la ville siège de l'exposition. L'exposition est une sorte de "ville dans la ville ", mais en même temps la grande ville, la métropole, est une sorte d'exposition permanente : c'est un lieu qui frappe l'imagination des ouvriers tout autant que l'exposition.

Du reste, bien qu'elle exalte certains caractères de la métropole, la citadelle de l'exposition est autonome et séparée. Elle est, en effet, un lieu " autre ", dans lequel se trouvent concentrés visiteurs et travailleurs, provenant de tous les coins du monde ; elle devient donc un lieu d'échange et de rencontre fondamental pour le monde ouvrier ${ }^{15}$. Les contacts entre ouvriers de différentes nations se créent au niveau symbolique, dans les grandes occasions des congrès ouvriers, mais aussi dans la réalité quotidienne, à travers la fréquentation des restaurants, hôtels et lieux de socialisation réservés aux travailleurs pendant leur séjour.

\section{Technique et progrès}

Déjà mise en avant dans les discours publics sur l'exposition, la notion de progrès, dont l'édification est une fonction majeure des expositions, compte parmi les termes les plus fréquents dans les récits

14 Sur l'idée de progrès au XIXe siècle, Gennaro Sasso, Tramonto di un mito: l'idea di uprogresso» fra Ottocento e Novecento, Bologne, il Mulino, 1984; Reinhart Koselleck, Christian Meier, Progresso, Venezia, Marsilio, 1995 (2e éd.) ; Pierre-André Taguieff, Le sens du progrès. Une approche historique et philosophique, Paris, Flammarion, 2004.

15 Pour une analyse du concept de lieu « autre » ou d'espace « autre », Michel Foucault, Eterotipia. Luoghi e non-luoghi metropolitani, Milan, Mimesis, 1994.

118 Documents pour l'histoire des techniques nº 18 - décembre 2009 
ouvriers. Les expositions établissent un rapport clair entre le progrès, le développement technologique et l'idée plus générale d'une " amelioration of mankind $\|^{16}$. Elles constituent l'un des moyens par lesquels passe, au cours du XIXe siècle, la construction culturelle d'une nouvelle exaltation de la technique et des inventions, des "Nouveaux Prométhées $\|^{17}$ qui remplacent le vieux Panthéon où siégeaient jadis les héros des batailles et de la politique, par un "Panthéon technologique " $^{18}$.

Devant le décor spectaculaire et fantasmagorique de l'exposition, expressément conçu pour fasciner et captiver l'émotion, les spectateurs ouvriers sont presque désarmés. La valeur implicite du message des expositions, véritable hymne au progrès, apparaît comme une constante dans les comptes rendus ouvriers pour toute la période considérée. Ce qui évolve dans le temps, ce sont les références, les symboliques et les représentations, quand, de la conception abstraite du progrès dans sa valeur plus générique et " politique ", on passe à l'examen des modalités effectives dans lesquelles il s'incarne et en particulier dans le rapport à la technique. Principal moyen d'affirmation du progrès, la technique provoque des réactions contrastées ${ }^{19}$. Alors que l'idée de progrès reste un fil conducteur du discours ouvrier, chaque technique peut être vue de façon plus ou moins critique ou changer de signification au cours du temps.

Un exemple emblématique est celui de l'énergie électrique ${ }^{20}$. Pour les travailleurs milanais à Paris en

16 « Memoirs of John McKie (c. 1820-1915) ॥, 5 vols., vol. III, p. 59, cité par Christine MacLeod, Heroes of invention. Technology, liberalism and British identity 1750-1914, Cambridge, Cambridge university press, 2007, p. 216.

17 C. Macleod, Heroes of invention, op.cit., pp. 27 et suiv. 18 lbid., pp. 181 et suiv.

19 Pour une analyse théorique du concept de technologie au XIX ${ }^{\circledR}$ siècle, Liliane Hilaire-Pérez, « La pièce et le geste. Entreprises, cultures opératoires et marchés à Londres au XVIII siècle ", mémoire inédit en vue de l'habilitation à diriger les recherches de I'Université Paris I-Panthéon-Sorbonne, sous la direction de Dominique Margairaz, décembre 2008, en particulier « La rationalité technologique, entre économie industrielle et économie du produit», pp. 1-52; plus en général sur le « commencement» de l'usage du terme, sur la définition du concept et sur l'entrée de la discipline dans l'univers scientifique, Jacques Guillerme et Jan Sebestik, "Les commencements de la technologie ", Thalès, 1968, xII, pp.1-72, réédité dans Documents pour I'histoire des techniques, 14, 2007, pp. 49-122 ; les résultats les plus récents de cette recherche ont été publiés dans un article en italien de Jacques Guillerme et Jan Sebestik, « Gli inizi della tecnologia ", Storia del disegno industriale, 1750-1850, Milan, Electa, 1989, pp. 14-37.

20 Sur le rôle de l'électricité aux expositions universelles,
1889, l'électricité représente l'une des attractions les plus extraordinaires de la ville et de l'exposition et elle justifie, dans leurs comptesrendus, un usage immodéré de superlatifs :

" A la tombée de la nuit, ce fut un émerveillement de voir l'arc de triomphe et la grande place de la Concorde entourés de jardins splendides et de très belles statues d'où s'écoulait l'eau. Mais le plus beau fut de voir le grand éclairage au gaz et la lumière électrique, dont l'effet fut splendide, en un mot il nous semblait être éclairé par la lumière du soleil $\gg^{21}$.

Même si, à l'exposition de 1889, l'électricité n'a pas encore une place autonome dans le circuit de l'exposition, avec ses jeux de lumières spectaculaires organisés pour l'occasion, elle représente non seulement le triomphe d'une technique extrêmement avancée pour l'époque, mais elle assume aussi un caractère métaphorique en tendant à matérialiser les valeurs les plus profondes et caractéristiques de l'idée de progrès. Ces valeurs assurent non seulement le plus grand bien-être, mais elles combattent et frappent les aspects négatifs et nuisibles de la tradition, identifiables dans la guerre et dans la violence. En ce sens, l'électricité acquiert une valeur quasi sacrée avec des inflexions millénaristes.

«L'ère des combats est finie [...] l'ère du travail est en plein essor [...] Le génie de la guerre doit fuir, aveuglé devant la splendeur de la lumière électrique, comme dans les tableaux mystiques du Moyen Âge le diable fuyait devant la Croix $11^{22}$.

Bernadette Bensaude-Vincent, « En flânant dans les expos : images de l'électricité "1, Culture technique, $n^{\circ}$ 17, mars 1987, pp. 89-93. Plus spécialement sur les expositions parisiennes, Patrice A. Carre, "Expositions et modernité : électricité et communication dans les expositions de 1867 à 1900 ॥, Romantisme, III, 1989, pp. 37-48; Fabienne Cardot, "L'éclair de la favorite ou l'électricité à l'Exposition de 1889 ॥, Le mouvement social, octobre-décembre 1989, n० 149, pp. 43-58.

21 Relazione del viaggio e del progresso all'Esposizione Internazionale di Parigi 1889 di Baroni Francesco della società dei lavoratori di Crema, Archives municipales de Milan [ensuite AM Milan], Esposizione di Parigi 1889, cart. 3 (Sul far della sera fu una meraviglia al vedere l'arco del trionfo e la gran piazza della Concordia circondata da stupendissimi giardini e di bellissime statue che tramandavano acqua. Ma il più bello al vedere fu la grande illuminazione a gaz, e la luce elettrica, che riuscì stupendissima, insomma ci sembrava di essere illuminati dal sole $॥)$.

22 Relazione della esposizione di Parigi 1889, dell'operaio 
Par la suite, la technique tend à devenir moins voyante, sa puissance devenant presque cachée et occulte, pendant qu'elle augmente évidemment d'intensité. Comme l'observe l'ouvrier mécanicien Settembre Cuppini, à Milan en 1906 :

« Dans le phénomène d'évolution, où tout change, même la caractéristique de l'exposition de Milan en a subi les effets. Dans les autres expositions, régnaient majestueusement, les machines motrices fixes à la vapeur, qui transmettaient vie et énergie à tout l'outillage principalement dans les galeries du travail : ici par contre tout est en mouvement, sous l'effet d'une puissance presque occulte. L'Électricité $12^{23}$.

\section{Technique et travail}

Si la technique a ce rôle central dans les représentations de l'idée de progrès, les évaluations des travailleurs ne se limitent pas à son apologie. Comme effet immédiat, la technique a une dimension spectaculaire, elle renferme les valeurs essentielles de l'idée de progrès; dans un deuxième temps, après une réflexion plus approfondie, en traduisant la substance « politique » de ce message sur un plan pratique et immédiatement accessible à l'expérience de travail des ouvriers, elle permet d'exprimer une série de résistances individuelles qui émergeraient difficilement à un niveau « idéologique » plus général.

C'est le cas du très large répertoire d'observations critiques adressées par les ouvriers sur le fonctionnement des machines. D'après leurs commentaires, les machines réussissent toujours à être plus rapides, constantes et économiques que la main de l'homme, mais elles produisent aussi en permanence des défauts, des imperfections, sans compter l'effet néfaste du

Caldara Antonio di Treviglio, ibid. (« l'era dei combattimenti è finita [...] l'era del lavoro è nel suo pieno sviluppo [...] Il genio della guerra deve fuggire abbacinato davanti allo splendore della luce elettrica, come nei quadri mistici del medio-evo il diavolo fuggiva davanti alla Croce »).

23 L'Esposizione di Milano. La scarsa mostra di macchine a vapore in genere. Relazione dell'operaio macchinista Settembre Cuppini della Società Tramw elettrici di Bologna, Archives nationales de Bologne [ensuite AN Bologne], Chambre de Commerce, V/141, Esposizione di Milano. (« Nel fenomeno di evoluzione, che tutto cambia, anche la caratteristica dell'Esposizione di Milano, ne ha subiti gli effetti. Nelle altre esposizioni, troneggiavano, le imponenti motrici fisse a vapore, che inducevano vita ed energia, a tutto l'altro macchinario principalmente nelle gallerie del lavoro: qui invece tutto è in moto, da una potenza quasi occulta. L'Elettricità ॥). chômage : la production de qualité est encore réservée au « métier » ouvrier, c'est-à-dire au travail des ouvriers qui utilisent encore des procédés liés aux compétences spécifiques et individuelles, souvent manuelles.

Les travailleurs parviennent souvent à discerner, sous les apparences éblouissantes des appareils de l'exposition, les défauts et les imperfections dont la pratique du métier leur permet de prendre conscience :

«Entre les innombrables volumes, opuscules et fascicules exposés, certains bien sûr étaient vraiment dignes d'éloges et beaucoup aussi de belle apparence ; mais [...] trop souvent l'habit ne fait pas le moine, et en soulevant ces vêtements voyants, ces petites couvertures aux couleurs vives, l'on découvrait tantôt un défaut, tantôt [...] des irrégularités de presse dues aux machines imparfaites et bien souvent à la négligence et à l'inexpérience de l'imprimeur [...] $11^{24}$.

Un ouvrier mécanicien milanais, Gaetano Colla, à Paris en 1900, observe à ce propos:

" C'est avec un vrai plaisir que j'ai [...] admiré le grand emporium de machines agricoles qui aujourd'hui devraient être l'aide la plus valable et la plus constante pour tous ceux dont la fatigue de la culture épuise les forces après peu de temps. J'ai dû toutefois déplorer à contre cœur que ces machines indiquées pour apporter une aide au bras de l'homme, une aide économique et matérielle, aient au contraire réussi, grâce à leur travail, à écraser, à rendre totalement inutile le bras de l'homme. Ainsi, alors que sans les machines travaillaient et vivaient naturellement vingt personnes, aujourd'hui leur aide puissante a divisé ce nombre par dix $12^{25}$.

24 Relazione di Paolo Contedini tipografo compositore. Relazioni degli operai romani inviati all'esposizione generale italiana di Torino nel 1884, Rome, Tipografia Editrice Romana, 1886 (" tra gli innumerevoli volumi, opuscoli, fascicoli esposti, ve n'erano certo, di veramente meritevoli d'encomio, e moltissimi anche di bella apparenza; ma [...] troppo spesso l'abito non fa il monaco, e sollevando quelle vesti appariscenti, quelle copertine dai colori vivaci, si scopriva ora un difetto, ora [...] irregolarità di stampa dovute alle macchine imperfette e bene spesso a trascuratezza e inesperienza dell'impressore »). 25 Relazione dell'operaio montatore meccanico Colla Gaetano all'Esposizione di Parigi del 1900, Archives historiques de la Chambre de Commerce de Milan, Esposizioni e Fiere - cart. 187 (« È con vera compiacenza che ho [...] ammirato il grande emporio di macchine agricole che dovrebbero essere oggi l'aiuto più valido più costante di coloro cui la fatica della 
Dans tous les cas, devant ou derrière chaque machine, il y a toujours un travailleur. Des comptes rendus ouvriers, il ressort que plus la technique est incorporée dans les machines, plus elle exige une évolution, une adaptation et un perfectionnement de la part du travailleur.

En outre, les travailleurs expriment fréquemment une ambivalence assez évidente entre l'acceptation des machines comme expression du progrès et la peur d'une baisse du travail, d'une menace pour l'emploi et leur niveau de vie ${ }^{26}$. Le discours est déterminé par une dialectique entre l'héroïsme du travail et en particulier du travail qualifié, qui s'est diffusée au niveau international dans la seconde moitié du XIXe siècle, et les développements techniques de la deuxième révolution industrielle qui tendent à réduire de plus en plus le rôle et l'habileté individuelle de l'ouvrier de métier. Le rapport entre l'habileté individuelle, la spécialisation de l'ouvrier et l'idéologie plus générale du progrès est confirmé par le fait que souvent les ouvriers contribuent, par leurs observations critiques, à l'amélioration des procédés productifs. II arrive fréquemment que des ouvriers envoyés comme observateurs à une exposition participent à d'autres expositions comme exposants, avec des produits ou des procédés techniques originaux, fruits de leur génie et de leur expérience professionnelle.

coltivazione rende impotenti dopo poco tempo. Ho dovuto però in cuor mio deplorare che queste macchine che sarebbero state indicate per un aiuto al braccio del vomo, aiuto economico e materiale siano invece riescite, mercé la loro professione, a schiacciare a rendere totalmente inutile il braccio dell'vomo. Di modo che mentre senza la macchina lavoravano venti persone e naturalmente vivevano, oggi coll'aiuto poderoso di esse quel numero ha dovuto decimarsi »). 26 Sur le rapport des ouvriers aux machines au XIX ${ }^{e}$ siècle, Maxine Berg, The machinery question and the making of political economy 1815-1848, Cambridge, Cambridge university press, 1980; Andrew Zimmerman, « The ideology of the machine and the spirit of the factory : remark on Babbage and Ure ॥, Cultural critique, 1997, n³7, pp. 5-29 ; C. MacLeod, Heroes of invention, op. cit. ; Vincent Bourdeau, François Jarrige, Julien Vincent, Les Luddites. Bris de machines, économie politique et histoire, Maison-Alfort, Editions Ère, 2006 ; pour le cas français, Michelle Perrot, « Les ouvriers et le machines en France dans la première moitie du XIX siècle ॥, Le soldat du travail, n. 32/33, septembre 1978, pp. 347-373 ; François Jarrige, « Les ouvriers parisiens et la question des machines au début de la monarchie de Juillet » dans ,Patrick Harismendy éd., La France des années 1830 et l'esprit de réforme, Rennes, Presses universitaires de Rennes, coll. Carnot, 2006, pp. 21 1-222 ; id., " Le martyre de Jacquard ou le mythe de l'inventeur héroïque (France XIXe siècle) ॥,Tracés. Revue de Sciences humaines, $n^{\circ} 16$, mai 2009, pp. 99-118; id. Face au Monstre mécanique. Une histoire des résistances à la technique, Paris, IMHO, 2009
Du reste, la conception des inventions comme nées de l'effort collectif de la multitude de travailleurs qui avaient contribué à créer les conditions qui avaient permis aux grands inventeurs de s'imposer au public, faisait partie intégrante de la critique radicale du mythe de l'inventeur-héros ${ }^{27}$. Même sur cette base de participation active à l'effort productif et au développement du progrès technique, les travailleurs cherchaient à rendre manifestes les instances de tutelle, de protection et de sauvegarde de certaines valeurs fondamentales telle la santé à l'usine. Andrea Biagini, un imprimeur romain à l'exposition nationale de Turin en 1884, écrit à propos de l'établissement italien de Nebiolo :

« Je restai extasié de voir environ vingt machines toutes en action... chaque machine est mise en marche par un ouvrier [...] ce qui mérite le plus d'éloges à l'usine c'est la manière dont elle a réorganiser ses locaux, en observant toutes les prescriptions hygiéniques pour protéger, autant que possible, les ouvriers des fréquentes maladies professionnelles auxquelles sont sujets les fondeurs de caractères d'imprimerie $11^{28}$.

\section{Identité ouvrière et fierté du métier}

Le métier est comparé aux progrès des processus productifs présentés aux expositions, mais il reste un point de référence indispensable.

Bien que les travailleurs envoyés aux expositionssoient classés sous des catégories simplifiées comme celles d' " ouvrier » ou d' « industrie », catégories qui renvoient à un système de production moderne et avancé, en réalité ce sont en grande majorité des travailleurs très qualifiés, des ouvriers de métier proches des véritables artisans, voire, dans le meilleur des cas, comparables aux factory artisans. Par conséquent, leur culture et leur identité professionnelle sont profondément enracinées dans une réalité productive qui subit de plein fouet l'impact innovateur de l'exposition. Les expositions semblent faites pour susciter ce mécanisme, entre le

27 Voir à ce propos les pages très significatives que Christine MacLeod consacre à Thomas Hodgskin, le premier analyste à avoir proposé une critique radicale à l'héroïsme des inventeurs, notamment du culte de James Watt; C. MacLeod, Heroes of invention, op. cit. pp. 155 et suiv.

28 Relazione di Andrea Biagini fonditore tipografo, op. cit. (« restai estatico e vedere circa venti macchine tutte in azione [...] ogni macchina è messa in azione da un operaio [...] ciò che più merita lode alla ditta è il modo con cui ha ridotto il locale, osservando tutte le prescrizioni igieniche onde salvare gli operai, per quanto è possibile, da quelle frequenti affezioni morbose cui vanno soggetti i fonditori di caratteri »). 


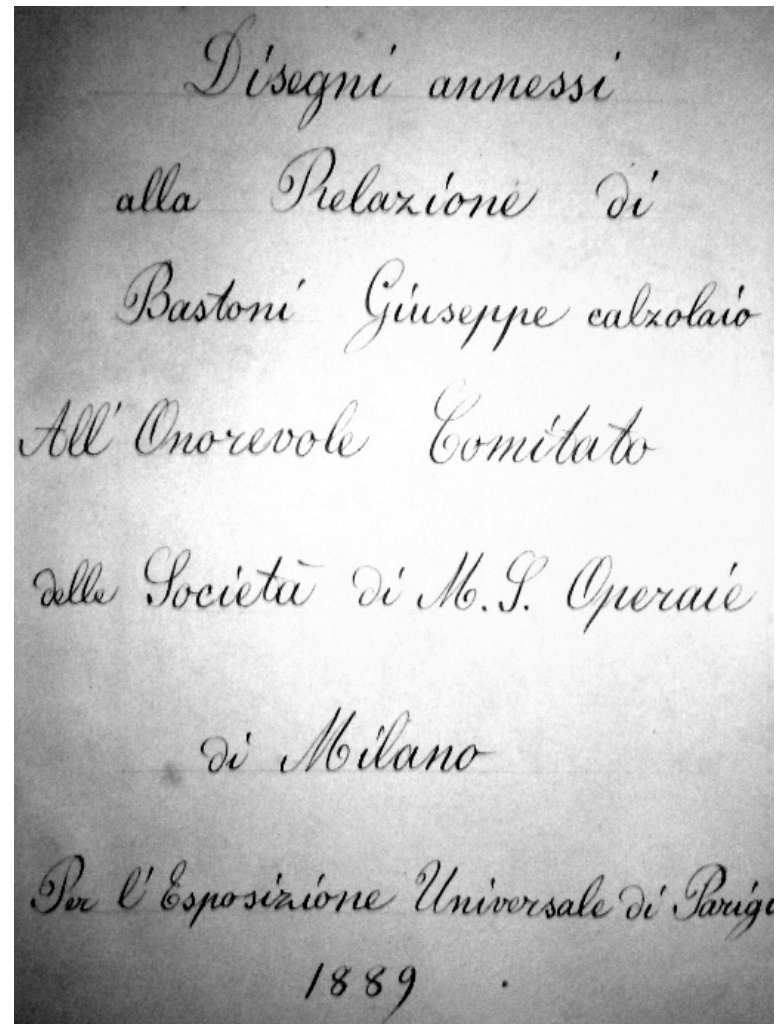

fig. 4 et 5 - Frontispice et dessins annexes au compte rendu de Bastoni Giuseppe cordonnier à l'exposition universelle de Paris 1889, Archivio civico del Comune di Milano, Esposizioni e Fiere, Esposizione di Parigi 1889, cart. n. 3.

milieu productif de provenance et les frontières des nouvelles techniques : à l'instar des ouvriers florentins envoyés à l'exposition de Milan en 1906, les ouvriers milanais qui se rendent aux expositions de Paris pendant la seconde moitié du XIX siècle sont confrontés à un contexte productif considérablement plus avancé qui contraste avec le leur.

Dans ces conditions, l'identité ouvrière est soumise à de fortes tensions, mais elle n'entre pas nécessairement en crise. Dans certains cas, elle paraît même renforcée ; dans d'autres, elle intègre l'innovation dans le cadre de valeurs et de conceptions préexistantes ; enfin, elle peut se mêler à des sentiments et des appartenances identitaires suggérés par l'événement de l'exposition, comme le nationalisme.

Le premier aspect concerne l'importance du métier et, plus généralement, le rôle du travailleur, de son habileté et de sa conscience professionnelle pour déterminer la qualité et le succès de la production. Les ouvriers observent que les machines tendent à marginaliser le métier et l'habileté professionnelle, l'apport « intelligent » de l'ouvrier à la production :

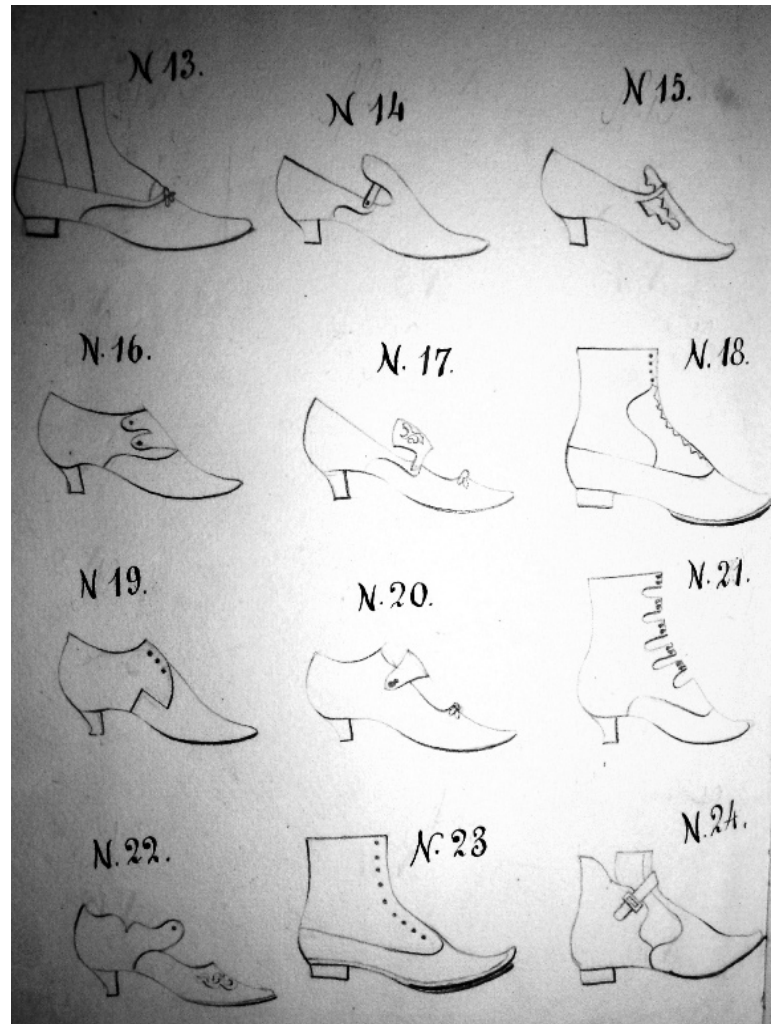

"L'United Shoe Machinery, compagnie des machines de chaussures [...] expose son (peutêtre trop) nombreux outillage [...] avec un tel outillage, on tend à exclure absolument du travail l'intelligence de l'ouvrier, elle ne reste qu'un accessoire $12^{29}$.

Bien que les ouvriers soient étonnés des potentialités des inventions, ils ne manquent pas d'en remarquer les défauts, qui se traduisent dans l'imperfection des produits. La qualité est encore un attribut exclusif du métier :

«cet outillage est d'une conception sublime mais je le crois encore imparfait [...] De la fabrication des formes en bois, à la coupe des peaux... au remplissage, au rognage, au planage, à la formation et au tréfilage des talons, au râpage, à l'estampillage, au ponçage, au lustrage, au nettoyage, tout est exécuté avec des types différents de machines, avec une rapidité prodigieuse, mais

29 Relazione della visita fatta all'esposizione di Milano dal lavorante calzolaio Romeo Persiani, AM Florence, Cerimonie, Festeggiamenti, Esposizioni, cart. 3, n. 5050 (« La United Shoe Machinery, compagnia delle macchine da scarpe [...] pone in mostra il suo, forse troppo, numeroso macchinario [...] con tale macchinario si tende assolutamente ad escludere dal lavoro la intelligenza dell'operaio, essa non rimane che un accessorio »).

122 Documents pour l'histoire des techniques $\mathrm{n}^{\circ} 18$ - décembre 2009 
pas avec exactitude, comme on observe dans les chaussures finies, certaines sont gâtées par des rayures ou des coupures aux empeignes, la finition est grossière et imparfaite ; l'ensemble de la chaussure qui est toujours un type unique n'offre pas cette souplesse élégante et svelte qu'on observe dans les autres modes de travail $11^{30}$.

Le second point a trait à la tension entre le métier traditionnel et les nouvelles formes d'organisation des processus de production, en liaison avec la division du travail et la spécialisation productive qui prolongent l'ancienne conception du métier.

Comme l'affirme l'ouvrier lithographe milanais, Enrico Leopardi, à Paris en 1889 :

«La perfection du travail donc [est donnée] par le soin apporté en toutes circonstances [...] il est vrai qu'en France et en Allemagne l'ouvrier se consacre surtout et presqu'exclusivement à une seule chose, de sorte qu'il y a des mécaniciens imprimeurs et des pressiers qui impriment sans arrêt les mêmes couleurs, qui se consacrent toujours à la même opération, pouvant ainsi acquérir une pratique spéciale et arriver à une parfaite connaissance des difficultés d'une matière donnée, alors que chez nous, l'ouvrier doit un peu tout faire, avec en plus des moyens trop économiques, qui ne permettent pas d'obtenir un résultat parfait du travail $\|^{31}$.

$30 \mathrm{lbid}$. (questo macchinario è di una sublime concezione ma lo credo ancora imperfetto [...] Dalla fabbricazione delle forme in legno, al taglio delle pelli [...] alla riempitura, raffilatura spianatura, formazione e trafilatura dei tacchi, alla raspatura marcatura, smerigliatura, lustratura, pulitura, tutto è eseguito con tipi differenti di macchine, con una rapidità prodigiosa, ma non con esattezza, come si osserva nelle calzature finite, alcune delle quali sono sciupate per fregature o tagliature ai tomai, la finitura è rozza e imperfetta; l'insieme della scarpa che è sempre un unico tipo non offre quella elasticità elegante e svelta come si osserva nelle altre lavorazioni »).

31 Relazione dell'operaio litografo Enrico Leopardi, AM Milan, Esposizioni e Fiere, Esposizione di Parigi 1889, cart. n. 3 (« La perfezione del lavoro dunque [è data] dall'accuratezza di tutte le circostanze [...] è ben vero che in Francia e in Germania l'operaio in genere si dedica specialmente e quasi esclusivamente ad una cosa sola, talché vi sono macchinist e torcolieri che stampano continuamente quei dati colori, cosicché dedicati sempre alla stessa operazione possono farsi una pratica speciale e venire a perfetta cognizione delle difficoltà di una data materia, mentre da noi, l'operaio deve fare un po' di tutto e di più con mezzi troppo economici, cioè non atti ad un perfetto risultato del lavoro $»$ ).
Le troisième aspectest que ces thèmes, intimement liés à la dimension technique du métier, se croisent avec une autre composante fondamentale de l'idée de progrès, liée à des valeurs sociales : celle d'une diminution constante, voire d'une disparition des formes de conflit, sur le plan tant social qu'international. Dans ce dernier registre, s'exprime le problème posé par l'ambiguité entre l'appartenance nationale des ouvriers et l'internationalisme pacifiste.

\section{Internationalisme, pacifisme et identité nationale}

Déjà en 1851, l'exposition du Crystal Palace entendait établir une « idéologie de la paix internationale et de la bonne volonté » (" ideology of international peace and goodwill $\gg)^{32}$, sous la houlette de l'Angleterre. Sur la base des principes du libre-échange et du libéralisme, cette puissance était capable d'instaurer une stratégie de collaboration entre les nations et les classes sociales, opposée aux conflits politiques et sociaux qui avaient caractérisé le contexte européen dans les années précédentes (1848-1849). La reine Victoria, dans son discours d'ouverture à l'exposition, avait clairement exprimé ce but pacifiste : «Mon vif désir de promouvoir parmi les nations la culture de tous ces arts qui sont engendrés par la paix et qui, à leur tour, contribuent au maintien de la paix dans le monde $\|^{33}$. Dans la culture populaire de l'époque, cette capacité du RoyaumeUni de recueillir, pour la première fois dans l'histoire, les efforts conjoints de toutes les nations du monde allait jusqu'à supposer une nouvelle "Sainte Alliance " "34 $^{34}$ entre les nations, cette fois-ci non pas avec un esprit politique d'orientation réactionnaire, mais avec des buts économiques inspirés par une philosophie libérale. La Grande-Bretagne était le pays naturellement désigné pour cette tâche parce qu'elle était :

« le seul pays libre d'Europe, à l'exception peutêtre de la Belgique et de la Hollande ; et cela, grâce à notre commerce qui s'étend si largement, à notre activité incessante, à notre richesse diffuse dans le monde entier, à nos bateaux à vapeur sur chaque mer, à nos lignes de communication allant jusqu'aux parties les plus reculée de la terre, et ainsi qu'à nos immenses triomphes mécaniques et scientifiques $11^{35}$.

32 C. MacLeod, Heroes of invention, op. cit. p. 215 33 « my anxious desire to promote among nations the cultivation of al those arts which are fostered by peace and which in their turn contribute to maintain the peace of the world ॥; P Greenhalgh, Ephemeral vistas, op. cit., p. 17. 34 " The old and new Holy Alliance ", The Illustrated London News, XVIII, 491, 7.6.1851.

35 « The only freecountry in Europe, Belgium and Holland 
Grâce à ces prérogatives, l'Empire anglais pouvait se proposer d'étendre aux autres pays, aux « nations qui travaillent et non qui se battent» (« working, and non fighting nations $»)$, de toute la terre, son propre modèle de développement qui liait étroitement pacifisme et progrès économique ${ }^{36}$.

Le discours pacifiste devient par la suite, de manière plus ou moins explicite, I'un des traits dominants de ces rencontres internationales ; parfois en négatif quand, par exemple, la date de certaines grandes expositions est déplacée pour éviter la coïncidence avec une guerre en cours. Le message explicitement pacifiste et d'opposition à la guerre n'est cependant pas toujours présent dans les comptes-rendus ouvriers; il se manifeste à certaines périodes ou occasions significatives, ou apparaît comme une référence obligée dans les commentaires sur les pavillons qui exposent des armes et des machines de guerre. Ainsi s'exprime un ouvrier bolognais en face du Pavillon de la Marine à Milan, en 1906 :

"Une quantité d'engins de guerre certes ingénieux et fabriqués à la perfection, mais toujours dominateurs et destructeurs de l'humanité et certainement ceux qui inventent et perfectionnent ces engins n'aspirent pas à concourir pour le prix Nobel de Suède qui fut, par sa dynamite, le plus meurtrier à nos jours et qui à sa disparition laissa un prix destiné à qui aurait trouvé la manière de ne plus utiliser sa poudre, c'est-à-dire de ne plus faire la guerre $)^{37}$.

Les ouvriers parlent souvent de "compétition ", ils utilisent des expressions comme " compétition internationale et paisible du travail » selon un modèle qui, comme il arrivera bientôt pour les Olympiades, peut concilier pacifisme et compétition nationale.

perhaps, excepted; and that, with our widely stretching commerce, our incessant activity, our wealth diffused in every part of the world, our steam-ships on every sea, our lines of communication to the remotest parts of the earth, no less than our great mechanical and scientific triumphs $»$; ibid. 36 P. Greenhalgh, Ephemeral vistas, op. cit. p. 18; J. A. Averbach, The Great Exhibition, op. cit. pp. 161-163.

37 Relazione dell'operaio Luigi Tagliavini, AN Bologne, Chambre de Commerce, V/141, Esposizione di Milano (« una quantità di ordigni benché ingegnosi e lavorati a perfezione, ma sempre prepotenti e distruttori dell'umanità e che certamente quelli che inventano e perfezionano questi ordigni non aspirano a concorrere al premio Nobel di Svezia che fu, mediante la sua dinamite, il più micidiale ai giorni nostri e che al suo morire lasciò un premio a chi avesse trovato il modo di non più adoperare la sua polvere ossia di non fare più la guerra »).
Le thème de l'appartenance nationale s'avère plus problématique et contrasté. Dès le début, les expositions se sont structurées comme une " compétition » entre les nations ${ }^{38}$. Même s'il s'agit d'une compétition paisible et progressive liée à l'idée que le libre-échange et le conflit des intérêts respectifs conduisent à stimuler la concurrence et l'émulation, en réalité la confrontation entre les domaines nationaux est très forte et la rivalité clairement ressentie. La comparaison entre les pays revêt un contenu proprement technique, et le caractère national et local des marchés et des appareils de production joue un rôle central. Les références à la situation des industries nationales abondent dans toutes les expositions internationales. Elles trouvent également leur place, de différentes manières, dans les expositions nationales.

Parfois, le constat du retard de l'industrie nationale ne s'accompagne pas d'une critique négative, mais d'une évaluation globalement positive de l'effort accompli par la nation arrivée tardivement dans la compétition internationale.

L'Italie avait réalisée, non sans mal, son unification politique en 1861, achevée ensuite par la prise de Rome, en 1870. Jusqu'en 1861, environ $80 \%$ du commerce des États italiens se faisait au dehors des frontières de la péninsule. La réalisation d'un marché national avec l'application d'un système douanier unique obligea une grande partie des économies régionales à une reconversion profonde. Les classes dirigeantes italiennes avaient en outre résolument choisi une politique de libre-échange, mais elles furent contraintes de l'abandonner en 1878 et 1887, car l'évolution des moyens de transport et du commerce mondial avait provoqué une baisse considérable des prix des produits agricoles et semiouvrés, typiques de l'économie italienne. La puissante vague d'émigration qui en suivit et la réorientation des politiques économiques, créaient d'énormes difficultés mais aussi des opportunités nouvelles pour l'industrie italienne qui, dans ces années, connut une situation très variable selon les secteurs ${ }^{39}$.

Du point de vue ouvrier, l'unification signifie aussi l'accomplissement d'une révolution libérale car tous

38 A. Mattelart, L'invention de la communication, op. cit, p. 139 ; Roberto Romano, « Le esposizioni industriali italiane. Linee di metodologia interpretativa "I, Società e Storia, 7, 1980, p. 224.

39 Les secteurs productifs en fort développement jusqu' aux années 1880, sont le textile et les activités liées aux infrastructures et aux services ; à partir des années 1890, la sidérurgie et surtout la mécanique prennent leur essor, auquel s'ajoutent d'importantes réalisations dans le secteur électrique. 
les États italiens (Piémont exclu), étaient des États absolutistes, dépourvus de système constitutionnel ${ }^{40}$. Dans les jugements des ouvriers, on trouve donc souvent soit la conscience du retard de l'appareil industriel national, soit celle des potentialités de développement et d'amélioration que la très jeune économie nationale peutespérer, commel'observent à Vienne en 1873 deux imprimeurs romains Federico Reina et Giovanni Perino :

«En jugeant dans l'ensemble les produits exposés et en tenant compte du fait que notre imprimerie est très peu représentée, il faut reconnaître que I'Italie est assez en retard par rapport aux autres nations en ce qui concerne la perfection de l'imprimerie. Mais vu les tristes conditions où l'Italie languissait depuis si longtemps, serait-il juste et équitable de la comparer aux autres nations et de considérer le résultat comme une condamnation par rapport aux autres nations... ? $1^{41}$.

En d'autres occasions, le retard national est justifié par une sorte d'aversion des industriels et des artisans plus qualifiés à participer aux expositions, de sorte que la représentation nationale peut apparaître moins flatteuse que son état réel. C'est ce que soutient le couturier Luigi Marroni à Paris en 1878 :

«En matière de couture, j'ai trouvé la France supérieure à toutes les autres nations; chaque petite chose était faite comme il se doit, avec propreté, exactitude et tout ce qu'il faut pour faire un beau travail...

L'Italie, par la quantité des travaux exposés, occupait pour ainsi dire la dernière ligne. Mais l'explication en est la suivante. Les couturiers italiens, spécialement ceux de Rome, je ne sais

40 Les associations ouvrières italiennes ont très souvent une claire orientation patriotique, ce qui change seulement quand l'option démocratique alors dominante est remplacée, aux débuts du XXe siècle, par une orientation socialiste.

41 Società fra gli operai tipografi, Relazione di Federico Reina e Giovanni Perino-compositore e macchinista tipografi, Relazioni degli operai italiani inviati dalle Società operaie romane all'Esposizione Universale di Vienna nel 1873 a spese del municipio, Rome, Stabilimento Tipografico Italiano, 1875 " Giudicando dal complesso dei lavori esposti e pure tenendo calcolo che la nostra tipografia è assai scarsamente rappresentata, è pur d'vopo riconoscere che l'Italia è assai al disotto delle alte nazioni per quanto riguarda la perfezione della stampa. Ma, considerando le tristi condizioni in cui da sì lungo tempo languiva I'Italia, sarebbe egli giusto ed equo metterla a confronto colle altre nazioni e ritenerne il risultato come una condanna colle altre nazioni ? ») pourquoi, n'exposent jamais leurs travaux dans aucune expositions; en effet le peu que j' ai vu à l'Exposition (qui à vrai dire ne nous fait pas beaucoup honneur) était des articles de boutique de confection et de couturiers de seconde classe. Et comme dans toutes les expositions concourent toujours les habituels exposants, je vous citerai ici un extrait de rapport fait par des délégués couturiers français, envoyés à Vienne à l'exposition de 1873.

Voilà ce qu'ils disent de nous : "tous les travaux de la Section italienne sont d'aspect lourd, et leur vue nous a surpris. II semble impossible qu'un pays si avancé dans les arts soit, dans notre industrie, arrivé à une telle décadence [...]. Si ce qui a été exposé sort des bonnes maisons de Florence et de Milan (ce que nous ne croyons pas) nous serions contraints à plaindre les Italiens obligés de porter des habits si peu en harmonie avec le doux climat dont ils jouissent ; climat qui, nous semble-t-il, devrait les autoriser aux fantaisies les plus originales et les plus légères, des fantaisies qu'à nous le besoin d'être couverts nous interdit" $11^{42}$.

42 Relazione di Luigi Marroni rappresentante della Società di Mutuo Soccorso e di miglioramento fra i sarti, Relazioni degli operai romani inviati all'esposizione universale di Parigi a spese del municipio e della camera di commercio-Raccolte per cura del Comitato operaio per l'invio dei rappresentanti le arti e i mestieri all'esposizione universale di Parigi 1878, Rome, Tipografia del Senato, 1879 (La Francia per lavori da sarto, I'ho trovata superiore a tutte le altre nazioni; ogni più piccola cosa era fatta come doveva essere, con pulizia, esattezza e quant'altro si richiede per fare un bel lavoro... L'Italia, in partita di lavori esposti, si può dire che occupava l'ultima riga Ma eccovi la spiegazione di questo. I sarti italiani, e specialmente i romani, non so se per noncuranza od altro, non espongono mai i loro lavori in alcuna Mostra; difatti quel poco che ho veduto all'Esposizione (che a dire il vero non ci fa molto onore) era roba di negozi di confezione e di sarti di seconda classe. E siccome in tutte le Esposizioni concorrono sempre i soliti espositori, vi voglio qui produrre un brano di relazione fatta dai delegati sarti francesi, inviati a Vienna all'Esposizione del 1873.

Ecco cosa dicono a nostro riguardo: " tutti i lavori della Sezione italiana sono d'aspetto pesante, e siamo rimasti sorpresi nel vederli. Sembra impossibile come mai un paese così avanzato nelle arti sia nella nostra industria arrivato a tale decadenza [...] se quello che è stato esposto esce dalle buone case di Firenze e di Milano (che non crediamo), noi saressimo [sic] costretti a compiangere gl'Italiani obbligati a portare dei vestiari così poco in armonia con il dolce clima che godono; clima che a noi sembra dovrebbe autorizzarli alle fantasie le più originali e le più leggiere, fantasie che a noi c'interdice il bisogno di star coperti' »). 
Le cliché du génie italien, entendu comme produit naturel d'une tradition artistique et culturelle qui puise ses racines dans le temps, est assez fréquent dans les comptes-rendus :

"L'Italie avec ses produits, dans notre art, comparée aux autres nations, se tient encore suffisamment pour conserver sa qualité [...] ; cette qualité ne vient pas du fait qu'en Italie les amateurs de notre art s'efforcent de progresser dans le perfectionnement et le développement de celuici... mais seulement du fait que nous avons eu la chance d'hériter, de nos anciens maîtres dans l'art, de tels monuments exemplaires... $11^{43}$.

Bien que de nombreux jugements soient critiques et déplorent le degré insuffisant d'avancement de l'industrie italienne par rapport à l'industrie étrangère, les comptes-rendus laissent aussi transparaître un sentiment prononcé de l'appartenance nationale.

Très souvent, pour compenser l'impact négatif d'une comparaison objective défavorable, on retrouve une série de justifications et de suggestions sur le plan technique, ainsi que l'affirmation explicite d'un orgueil national. D'aucuns relèvent même avec fierté une avance de l'Italie par rapport aux autres nations, comme s'exprime un ouvrier de Monza, Imardo Crippa, à Paris en 1900 :

"La France, dans l'art des chapeaux mérinos produits à la machine, est très en retard par rapport à l'Italie, du fait que ces machines sont encore celles qui en Italie, spécialement à Monza, étaient utilisées au début, quand la mécanique commençait à pénétrer dans cet art, c'est-à-dire vers 1883. Les machines présentées [...] ne proposent aucune nouveauté, en ceci que la presse est encore réchauffée [à] la forme à gaz, la pression est donnée au chapeau de gomme avec une pompe manuelle, tandis que chez nous les formes sont réchauffées à la vapeur... $11^{44}$.

43 Relazione del Rappresentante della Società dei Marmisti Agostino Guidi, op.cit. (" L'Italia coi suoi prodotti, nella nostra arte, al confronto delle altre nazioni, ancora si sostiene quanto basti per mantenere il suo decoro [questo] decoro non deriva perché in Italia i cultori della nostra arte si studiano progredire nel perfezionamento e sviluppo di essa [...] ma unicamente perché abbiamo noi avuto la fortuna di ereditare dai nostri antichi maestri nell'arte tali monumenti ed esemplari »).

44 Relazione dell'operaio Imardo Crippa di Monza - Industria Abbigliamento e affini, Archives historiques de la Chambre de Commerce de Milan, Esposizioni e Fiere, cart. 187 (« la Francia nell'arte dei cappelli merinos prodotti colle macchine è
Le seul fait d'être à l'étranger, d'entrer en contact avec des réalités et des expériences différentes, ouvrait l'esprit des travailleurs à une comparaison entre la réalité qu'ils côtoyaient chez eux et ces lieux symboles de la modernité.

\section{La rencontre avec la métropole}

Pour les ouvriers, la visite de l'exposition représente une expérience extraordinaire car elle équivaut presque toujours à rencontrer un contexte urbain et productif plus avancé que celui d'origine. Le voyage en lui-même constitue une expérience qui reste profondément gravée dans I' "esprit du visiteur ". Cette expression utilisée par l'ouvrier florentin, fondeur d'objets d'art, Giulio Niccoli45, renvoie pour nous qui la lisons aujourd'hui au titre d'un livre de référence en la matière : The mind of the traveler d'Eric Leed ${ }^{46}$. Elle suggère en effet l'incidence de la rencontre avec une réalité parfois très différente sur l'état mental et sur la perception des ouvriers.

Parce qu'il les éloigne de la réalité quotidienne du travail, parce qu'il représente une expérience collective, le voyage est aussi une fête. L'amusement, la suspension des règles quotidiennes, le degré de liberté habituellement prévu par tous ces voyages laissent transparaître des éléments utiles pour étudier la mentalité ouvrière dans un sens plus général.

Finalement, par delà le parcours physique effectué par ces ouvriers de Florence à Milan ou de Milan à Paris, les comptes rendus font part d'un voyage d'un autre genre : un voyage mental à l'intérieur de l'exposition, vue comme un monde virtuel à parcourir et à explorer. Comme le note Alexander Geppert :

« Dans l'ensemble, les expositions montraient un 'temps gelé' et des lieux lointains dans un paysage urbain, en donnant aux visiteurs l'illusion que s'ouvraient d'inimaginables occasions de voyage dans le temps et dans l'espace $n^{47}$.

di molto in arretrato dell'Italia, inquantochè quelle macchine sono ancora quelle che in Italia e specialmente a Monza si adoperavano nei primi tempi in cui la meccanica cominciava a penetrare in quest'arte e cioè circa l'anno 1883. In queste macchine presentate [...] non vi sono nessuna novità, inquantochè la presse è ancora riscaldata [ill.] la forma a gaz, la pressione è data al cappello di gomma con una pompa a mano, mentre da noi le forme sono riscaldate a vapore $»)$. 45 Note appunti e impressioni dell'Esposizione di Milano. Relazione dell'operaio Giulio Niccoli, fonditore in oggetti di Belle Arti, AM Florence, Cerimonie, Festeggiamenti, Esposizioni, cart. 3, n. 5050. 46 Eric J. Leed, La mente del viaggiatore, II Mulino, Bologne, 1992, p. 259.

47 Alexander C.T., Geppert, « Luoghi, città, prospettive: le

126 Documents pour l'histoire des techniques $\mathrm{n}^{\circ} 18$ - décembre 2009 
La dimension spectaculaire du rêve, de l'arrivée dans ce que nos ouvriers définissent comme un " monde nouveau ", fait de "châteaux féeriques" et de "royaumes enchantés", se retrouve dans nombre de comptes-rendus. Tout aussi présente est la ville réelle - opposée à celle, fictive et éphémère, de l'exposition - dont les ouvriers perçoivent la valeur " révolutionnaire » et progressive synthétisée dans un deuxième temps par l'exposition et par les valeurs qu'elle exprime.

Une dimension importante des rapports de ces ouvriers réside donc dans la dialectique entre la ville réelle et la citadelle de l'exposition. Des études récentes ont mis en évidence que la métropole, siège de l'exposition, constitue une exposition " actuelle » ou « réelle », par opposition à l'exposition, "fictive ", momentanée et éphémère. De la même manière que les expositions sont considérées comme une "ville à l'intérieur de la ville ", les villes qui les accueillent peuvent elles aussi être considérées comme des expositions permanentes à rapprocher de l'exposition transitoire. À l'occasion de l'exposition universelle de 1889, la Pall Mall Gazette écrit :

«il y a beaucoup de choses à voir à Paris, mais la plus importante est Paris même. Paris vaut plus qu'une exposition quelconque. Toutes les expositions sont structurées plus ou moins sur le même modèle. Paris est par contre unique. Si les expositions sont plusieurs, Paris n'est qu'une seule. Paris est donc la première chose à voir, avant d'aller à l'exposition $\|^{48}$.

Ces sources rendent donc compte de l'image " exceptionnelle " de la ville, telle qu'elle apparaît aux yeux de ceux qu'on peut appeler des « touristes ouvriers ". Ceux-ci ne sont pas plongés, comme des touristes ordinaires, dans la vie quotidienne. La ville qu'ils visitent est une ville qui s'habille, qui se maquille pour l'occasion, qui affiche un visage extraordinaire, tout comme est extraordinaire la condition de ces travailleurs. Tout se passe comme si la ville offrait un spectacle à ces travailleurs quise rendaient pour la première fois au théâtre.

Les ouvriers se rendent bien compte de ce caractère exceptionnel; ils savent que la ville qu'ils visitent se présente à leurs yeux à un moment bien précis, celui de son plus grand triomphe, comme l'écrit le dessinateur milanais de tissus Antonio Parazzoli à l'occasion de son voyage à Paris en 1889 :

esposizioni e l'urbanistica fin de siècle ", Memoria e ricerca, $n^{\circ} 12$, a. XI, 2003, p. 129, pp. 115-136.

48 " Paris and its Exhibitions 》, Pall Mall Gazette Extra, 49, 26 juillet 1889, Londres, Pall Mall Gazette, 1889, p. 10 ; A. C.T. Geppert, Luoghi, città, prospettive, op. cit., p. 132.
« Paris !... Paris !...

Le rêve de mes nuits, l'éternel cauchemar de mon esprit, la pensée d'une partie de mon existence, je t'ai vue enfin...!

Je t'ai vue, ô belle et historique ville mondiale, je t'ai vue dans le moment, des plus solennels, celui de ton plus grand triomphe!

Je t'ai vue dans l'instant où, on peut le dire superbement, les Sciences ont atteint leur apogée ! $1^{49}$

Le caractère exceptionnel de la ville relève également de la capacité de celle-ci à exprimer l'excellence. Paris, dans ce cas puise dans l'extraordinaire pour inventer une nouvelle quotidienneté, différente, «moderne » : en témoigne par exemple la monumentalité de l'exposition, qui souvent survit à l'événement qui l'a créée, et devient un élément constitutif et symbolique de la ville.

Des comptes rendus entiers sont consacrés à l'impact de rencontre-collision et d'attractionrépulsion créé par la métropole de l'exposition. Milan pour les ouvriers florentins, comme Paris pour les ouvriers milanais, suscite des impressions plus ou moins équivalentes. La visite fournit l'occasion de comparer l'image préconçue, sur la base de connaissances indirectes ou stéréotypées, avec une expérience concrète, parfois déconcertante, voire abrutissante :

" Arriver à Paris la grande ville cosmopolite par excellence dont j'avais beaucoup lu et entendu parler, dont je m'étais fait dans la tête une idée que la réalité a de très loin dépassée ; en elle il y a le mouvement, la vie, enfin elle représente bien l'esprit agité et indompté de la race française. Tous les peuples y sont représentés, les jaunes, les noirs avec leurs coutumes bizarres se rencontrent à chaque pas ; bâtiments et immeubles majestueux noircis par les temps, mémoires et monuments historiques, voies larges et spacieuses et intérieurs nobles, un mouvement qui fait perdre la tête, chariots, voitures, omnibus, tramways à vapeur et électriques, chemins de fer aériens, bicyclettes et automobiles, quelque chose enfin qui semble impossible et qui semble un rêve ; la Seine

49 Relazione di Enrico Antonio Parazzoli disegnatore di tessuti, AM Milan, Esposizione di Parigi 1889, cart. 3 (« Parigi !...Parigi !... Il sogno delle mie notti, l'eterno incubo della mia mente, il pensiero d'una parte della mia esistenza finalmente ti vidi...! Ti vidi o bella e storica città mondiale, ti vidi in un momento dei più solenni, quello del tuo maggior trionfo! Ti vidi nell' 'istante che superbamente può dirsi, che le Scienze hanno raggiunto il loro apogeo! ॥). 
avec ses bateaux à vapeur rend la ville encore plus agréable $\|^{50}$.

« Nous ne connaissions Milan que par la description que des amis nous en avaient faite, on la savait une ville grandiose, moderne et belle et nous l'appréciions pour l'élan avec lequel elle répondait à chaque initiative, qu'elle soit commerciale, scientifique, artistique ou patriotique, mais l'idée que nous nous en étions faite était de beaucoup inférieure à l'impression laissée par la visite. La monumentale Cathédrale avec ses innombrables coupoles, la galerie grandiose, les grandes places, les voies spacieuses où s'agitait continuellement une foule cosmopolite et se succédaient des trams, des charrettes, des voitures qui enchantent et étourdissent. Les grands ateliers desquels l'on voyait entrer et sortir une foule d'ouvriers, tout est gravé dans notre esprit, tout constitue un souvenir heureux et inoubliable $n^{51}$.

En outre, dans la majorité des cas, le voyage se présente sous la forme d'une marche vers le "Progrès ». C'est le cas des ouvriers de Florence, de Bologne et de Rome envoyés à Milan ou à Turin, mais c'est aussi celui des ouvriers de Milan ou de ceux

50 Relazione della visita fatta all'Esposizione di Parigi nell'agosto 1900 da Colombo Rodolfo macchinista litografo, Archives historiques de la Chambre de Commerce de Milan, Esposizioni e Fiere, cart. 187 (« arrivare a Parigi la grande città cosmopolita per eccellenza di cui tanto lessi e sentii parlare di cui mi ero fatto nella testa un concetto che la realtà ha di molto superato; in essa vi è il moto, la vita, insomma ben rappresenta lo spirito irrequieto ed indomito della razza (corsivo mio) francese. Tutti i popoli in essa vi sono rappresentati, il giallo, il nero coi loro bizzarri costumi ad ogni passo si incontrano; edifici e palazzi maestosi anneriti dal tempo, memorie e monumenti storici, vie larghe e spaziose ed interni nobili, un via vai che fa perdere la testa, carri, carrozze, omnibus, tranvai a vapore ed elettrici ferrovie aeree, biciclette ed automobili, qualchecosa [sic] insoma [sic]che sembra impossibile e che par di sognare; la Senna coi suoi battelli a vapore rende ancor più piacevole la città »).

51 Relazione degli operai Umberto Zocca e Roberto (" Conoscevamo Milano solo per la descrizione avutane da amici, la sapevano città grandiosa moderna bella e l'apprezzavamo per lo slancio con cui rispondeva ad ogni iniziativa fosse commerciale o scientifica artistica o patriottica, ma il concetto che nella mete nostra ci eravamo formati era di gran lunga inferiore all'impressione ricevuta della visita testé fatta. II monumentale duomo dalle numerosissime cuppole [sic]la grandiosa galleria le grandi piazze, le vie spaziose dove è un agitarsi continuo di una folla cosmopolita e un succedersi di tranvai di carrozze di carri di automobili che incanta e stordisce. I grandi opifici dai quali si vede entrare e uscir schiere di operai tutto è fisso nella nostra mente, tutto costituisce un lieto ed indimenticabile ricordo ») de Turin envoyés à Paris (tout comme l'avait été le parcours Paris/Londres, organisé par Napoléon III pour des ouvriers français en 1862). II s'agit de faire prendre la mesure d'un retard et conscience du besoin de le rattraper. Un retard qui, pour les ouvriers italiens, à Paris comme à Milan, ne s'exprime pas seulement du point de vue économique mais aussi et surtout d'un point de vue politique, culturel et social sur lequel les stéréotypes nationaux d'autre part, ont une considérable influence. L'ouvrier mécanicien milanais Gaetano Colla à Paris en 1900, après avoir exposé ses impressions monumentales et artistiques sur les villes lumière, continue ainsi :

« Impression qui grandit quand je pense à la liberté dont on y jouit, liberté qui, bien que décrite par certains comme limitée, s'avère bien différente de ce à quoi je m'attendais ; considérablement supérieure à celle dont on peut jouir sur notre beau sol d'Italie. Dans mon bref séjour à Paris, il ne me fut jamais donné de voir des gardes municipaux, ni de sécurité publique [sic] se promenant à travers la ville avec des revolvers à la ceinture comme chez nous - comme si nous étions dans un état de brigands ou en état permanent de siège - et ceci dénote un remarquable degré de civilisation ; je n'ai jamais eu l'occasion de voir cette austérité affichée entre supérieurs et subalternes de la grande armée, telle qu'on l'enregistre chez nous; je n'ai jamais eu l'occasion d'observer, dans les lieux publics de rencontre, cette aristocratie dominante, le riche qui refuse le contact du travailleur, comme la majorité le pratique chez nous : mais par contre cette fraternité réciproque, cette communion de sentiments comme il convient à un peuple civil et vraiment libre $\bigwedge^{52}$.

52 Relazione dell'operaio montatore meccanico Colla Gaetano all'Esposizione di Parigi del 1900, Archives historiques de la Chambre de Commerce de Milan, Esposizioni e Fiere, cart. 187 (« Impressione che si ingrandisce allorché penso alla libertà che vi gode, libertà che per quanto taluni vogliano figurarla limitata, più del suo essere, io la riscontrai ben diversa di quello che mi aspettavo; di gran lunga superiore a quella che si gode nel nostro bel suolo d'Italia. Nel breve mio soggiorno a Parigi non mai mi fu dato vedere delle guardie di città, ne [sic] di sicurezza pubblica vagando per la città con tanto di revolver alla cintola come da noi, quasi si fosse in uno stato di briganti o in permanente stato d'assedio, e ciò denota maggiore civiltà; non mai mi fu dato vedere quel austerità palese fra superiori e subalterni del grande esercito, come si riscontra da noi, non mai mi fu dato vedere nei pubblici ritrovi quell'aristocrazia dominante , cioè che il ricco scansi il contato [sic] del lavoratore come si usa dalla maggioranza da noi; ma bensì quella reciproca 
Son collègue, Rodolfo Colomb, s'exprime avec le même enthousiasme :

" en France, [...] bien que beaucoup de monde soutienne qu'il n'y a pas de liberté, le travailleur peut faire entendre sa voix bien mieux que chez nous [...]. Les autorités traitent bien différemment les Bourses du Travail, les syndicats, les ligues, etc., alors que chez nous elles sont regardées comme des abris de malfaiteurs et de fauteurs de troubles [...]. L'organisation ouvrière forte est donc le bien-être du travailleur, et partout l'on s'en aperçoit en parcourant les galeries interminables de l'exposition $\|^{53}$.

Plus de vingt ans plus tôt, la situation apparaît similaire quand Annibale Renzi, ouvrier romain envoyé à l'exposition de 1878, argumente ainsi :

« Dans le petit bout de France parcouru, je ne faisais qu'apercevoir de chaque côté du chemin de fer des villages, des petites villes, des pays manufacturiers de toutes sortes et des industries. Si ce peuple se vante d'être grand, il l'est de fait, s'il se dit uni, il suffit de regarder leurs grandes associations manufacturières et commerciales qui envahissent la France, tout comme la majeure partie du monde, où ils font de grandes expositions de leurs produits, pour que tous les autres peuples en soient convaincus. Nous autres Italiens, surtout de cette ville, nous ne devons pas nous croire riches de grands capitaux, quand ces derniers restent enterrés ou servent seulement à alimenter la classe monopoliste qui chaque jour s'accroît davantage, au détriment des travaux et des industries qui découragent d'étudier et de travailler pour progresser, afin de pouvoir être aux côtés des autres nations $11^{54}$.

fratellanza quella comunione d'affetti come s'adice [sic] ad un popolo civile e veramente libero $॥$.

53 Relazione della visita fatta all'Esposizione di Parigi nell'agosto 1900 da Colombo Rodolfo macchinista litografo, op. cit. ("I in Francia [...] benché molti sostengono non vi sia libertà, pure il lavoratore può meglio che da noi far sentire la sua voce [...] Quanto diversamente sono trattate dalle autorità le Borse de lavoro, i sindacati, le leghe ecc. mentre da noi si guardano come covi di malviventi e di turbolenti [...] La forte organizzazione operaia è quindi il benessere del lavoratore, e dovunque lo si riscontra girando le interminabili gallerie dell'esposizione $॥$ ). 54 Relazione del rappresentante della Società di Mutuo Soccorso dei falegnami ed ebanisti di Roma, op. cit. (" nel breve tratto percorso della Francia non facevo che scorgere da ogni lato del cammino ferroviario villaggi, piccole città e paesi manifatturieri di varie specie e industrie. Se quel popolo si vanta
Ces dernières impressions montrent clairement la tentative récurrente des travailleurs pour replacer les expériences vécues dans un cadre de référence cohérent, afin si possible de confirmer et de consolider leur propre sentiment d'identité, notamment politique.

\section{Conclusion}

Les comptes rendus des travailleurs envoyés aux expositions représentent un véritable "genre " littéraire, limité dans le temps et dans l'espace. Comme l'observait Madeleine Rebérioux, vers la fin du XIX siècle pour la France (et, dans le cas italien, deux décennies plus tard), ces comptes-rendus perdent peu à peu leur importance et leur sens ${ }^{55}$.

Ces écrits s'inscrivent dans un moment de forte croissance du mode de production capitaliste qui exige l'emploi d'une main-d'œuvre toujours plus nombreuse, avec l'élaboration de cultures et de rhétoriques qui tendent à définir, de manière inédite, l'identité et la place hiérarchique des travailleurs industriels dans la société moderne.

Cette nouvelle classe sociale se définit comme une classe mobile, composite et changeante : l'évolution même des techniques modifie en permanence les processus de production et donc les positions des travailleurs au sein du système productif. Dans toutes les études les plus importantes sur le monde du travail, les thèmes de la culture professionnelle, de la persistance de la tradition face aux innovations supportées par les entreprises s'avèrent à la fois déterminants et difficiles à cerner avec précision, en raison du manque de sources directes susceptibles de témoigner de la richesse et de la complexité d'une telle transition.

Parce qu'ils s'insèrent dans un contexte linguistique et discursif complexe, les comptes-rendus des délégations ouvrières italiennes aux expositions universelles constituent une source essentielle pour révéler les processus à travers lesquels les travailleurs

di essere grande, lo è di fatto, se si dice unito, basta guardare le loro grandi associazioni manifatturiere e commerciali che invadono la Francia tutta come anche nella maggior parte del mondo, dove fanno le grandi esposizioni dei loro prodotti, perché ogni altro popolo se ne persuada. Noi italiani, ed in specie di questa città non dobbiamo ritenerci ricchi di grandi capitali, quando sia l'uno che l'altro, o restano sepolti o soltanto per alimentare la classe monopolista che in ogni giorno va prendendo larghe proporzioni, a danno delle opere e delle industrie che tolgono il coraggio di studiare e lavorare per progredire, onde potere essere al fianco delle altre nazioni $»)$. 55 M. Rebérioux, "Au tournant des expos ", op.cit., p. 8 ; id., " Les ouvriers et les expositions universelles de Paris au XIXe siècle ॥, op. cit. p. 199. 
construisent leur propre univers de valeurs, en liaison avec leur identité professionnelle et sociale, et la manière dont ils parviennent ainsi à donner du sens au difficile passage vers la «modernité ».

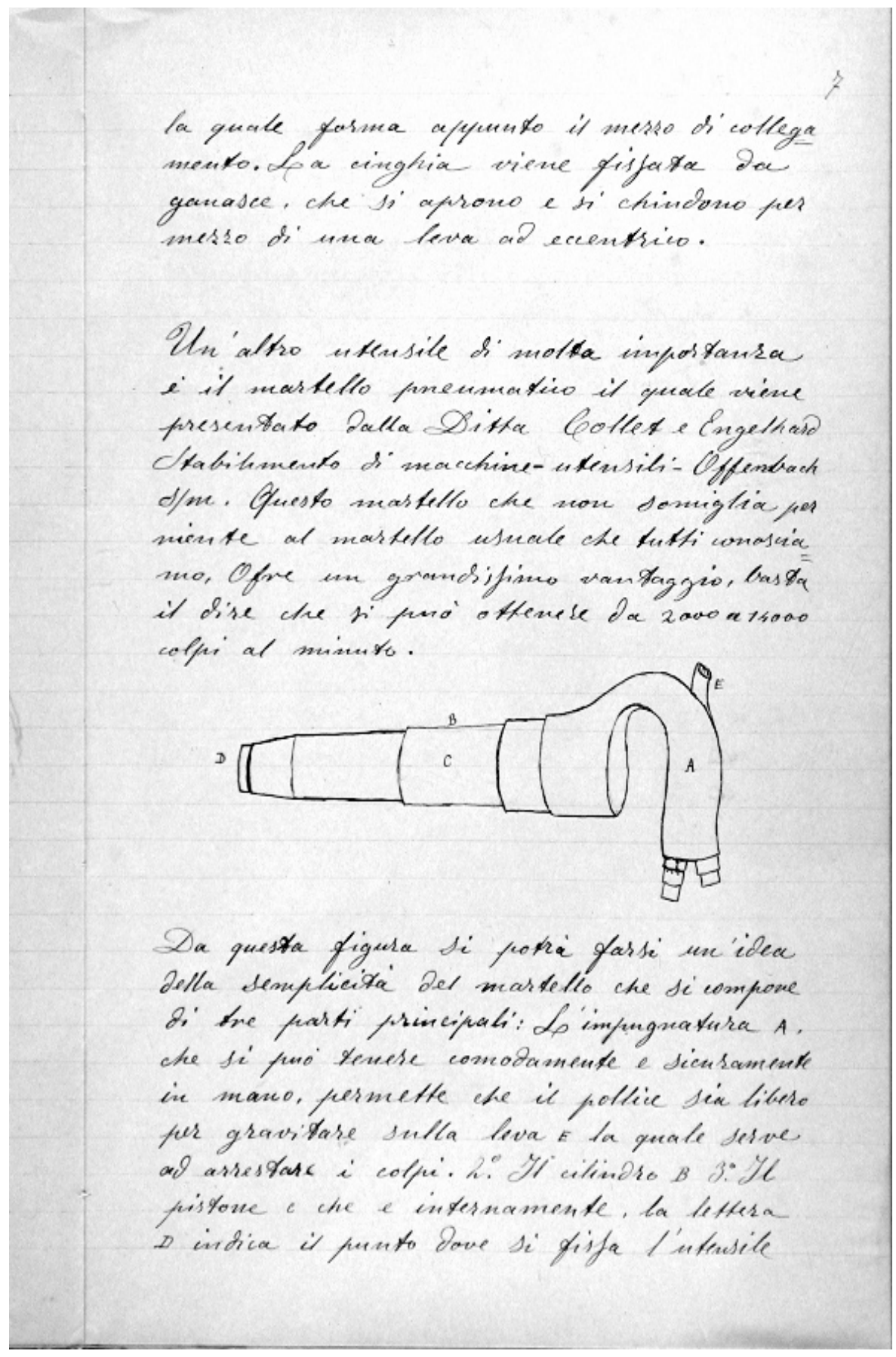

fig. 6 - une page du compte rendu de l'ouvrier mécanicien horloger Menaldo Augustino à l'exposition de Milan en 1906, ASCF, Comune di Firenze, Cerimonie, Festeggiamenti, Esposizioni, cart. 3, n. 5050. 